\title{
ENFERMEDAD EN ORÁN: EL DEVENIR-ANIMAL EN LA PESTE, DE ALBERT CAMUS
}

\author{
Melanie Moreira Abad ${ }^{1}$
}

\begin{abstract}
Resumen: En La peste, Albert Camus propone una metáfora que nos recuerda a la guerra. Esta misma metáfora puede ser pensada como un devenir-animal que resulta en un contagio global. La enfermedad que ataca a Orán no es la de la peste en sí misma, sino más bien el devenir que se contagia y que forma multiplicidades a través de las desterritorializaciones del ser hasta que este se transforma molecularmente y exterioriza un devenir-rata. El escenario que permite el devenir en Orán es el de la guerra, y esta guerra se escapa del plano de la ficción y nos hace pensar en cómo nuestra realidad está llena de esta peste que nos hace devenir-ratas. El hombre que pelea en la guerra empieza un proceso de deshumanización que atraviesa todos los devenires, pasando por el devenir-animal, para luego contagiar a los demás cuerpos a su alrededor. Esta peste que se contagia no es otra que el escenario que permite que los deveniresanimales sucedan. Guerra y peste resultan ser lo mismo en la medida en que hombres y ratas lo son también: cada uno es en sí mismo al mismo tiempo que lo es en el otro. Esta dinámica de deshumanización que conecta a los hombres de Orán con un otro animal, un otro rata, nos hace pensar en nuestras propias guerras, es decir, en nuestras pestes y en nuestros devenires-ratas: La peste es un relato que nos ubica frente a nuestro propio contagio colectivo.
\end{abstract}

\section{Enfermedad en Orán: el devenir-animal en La peste, de Albert Camus}

(...) y puede llegar un día en que la peste, para desgracia y enseñanza de los hombres, despierte a sus ratas y las mande a morir en una ciudad dichosa.

Albert Camus, en La peste

\section{¿O bien la multiplicidad que nos fascina ya está en relación con una multiplicidad que nos habita por dentro? \\ Gilles Deleuze y Félix Guattari, en Mil mesetas}

\begin{abstract}
Albert Camus (1913-1960) fue escritor argelino-francés que desarrolló, a partir de la obra de Franz Kafka, la filosofía del absurdismo. Una de sus obras más conocidas es La peste, publicada en 1947. En esta obra, Camus narra la historia de Orán en un año indefinido; en el pueblo de Orán las personas se han visto atrapadas por la monotonía y la indiferencia, y sus vidas parecen transcurrir sin que les importe. Esta monotonía se ve afectada cuando llegan unas ratas a la ciudad, y aunque inicialmente las personas deciden ignorar este hecho, las ratas poco a poco se van multiplicando hasta que la peste y la muerte son inevitables.

Albert Camus, Nobel de Literatura en 1957, nace en Argelia en 1913, cuando esta era colonia francesa, y vive la ocupación alemana en Francia durante la Segunda guerra mundial. Con una vida marcada por conflictos bélicos, Camus relaciona constantemente su obra con los comportamientos humanos adoptados en este tipo de situaciones, esto ocurre también en La peste.

La peste (1947), desde la lectura más obvia y al mismo tiempo más simbólica, constituye una alegoría a la guerra y a las consecuencias de esta, pero no solamente es eso, sino que
\end{abstract}

\footnotetext{
${ }^{1}$ Escuela de Literatura de la Universidad de las Artes, Guayaquil-Ecuador. E-mail: melanie.moreira@uartes.edu.ec.
} 
también representa el punto más alto de la filosofía humanista que Camus defendía²: la peste destruye, pero también supone la posibilidad de humanización, de heroicidad colectiva.

No obstante, La peste está llena de deshumanización también, pero en un sentido deleuziano; es decir, en la medida en que esta deshumanización significa la desterritorialización del ser: un devenir. Es así que esta novela que se apega más a la realidad que a la ficción, desde distintas lecturas, puede parecer que se presenta a través de una metáfora; sin embargo, es posible también establecer un diálogo con el concepto de devenir menor planteado por Gilles Deleuze y Félix Guattari -específicamente, el devenir-animal-.

Una de las primeras cuestiones significativas que surgen para el análisis de este deveniranimal es que se trate de una obra totalmente masculina: ningún personaje femenino alcanza la posición de personaje como tal. Algunas mujeres son mencionadas por los hombres cuando dicen que las extrañan, pero ninguna de ellas trasciende mayormente. Casi retratando a la guerra como una lucha de poder masculino en la que únicamente los hombres toman partida. Esto es fundamental al momento de hablar del devenir, ya que el hombre elimina su condición de hombre para deveniranimal, para devenir menor; no es una mujer la que deviene, sino el hombre.

Hemos visto cómo el hombre de guerra, por su furor y su celeridad, estaba atrapado en devenires-animales irresistibles. Estos devenires encuentran su condición en el devenir-mujer del guerrero. (Mil mesetas, p. 279 y 280)

Deleuze y Guattari toman a Jean-Pierre Vernant ${ }^{3}$ en esta cita para explicar que el hombre de la guerra ha atravesado un devenir que pasa por el devenir-animal, el mismo que en Camus es el medio para que se dé la peste. La peste en este caso es una condición que se le atañe solamente a los hombres, como proponiendo una ecuación en la que hombre da igual a peste, a guerra, y en esta entonces se produce el devenir donde los hombres primero devienen-mujeres, que es el primero de todos los devenires menores.

Si el devenir-mujer es el primer cuanto segmento molecular, y luego vienen los devenires animales que se encadenan con él, ¿hacia dónde se precipitan todos ellos? Sin duda, hacia un devenir-imperceptible. Lo imperceptible es el final inmanente del devenir, su fórmula cósmica. (Mil mesetas, p. 280)

Los hombres de Orán se encaminan hacia un devenir imperceptible: ha ocurrido algo dentro de ellos que los ha cambiado; se han desprendido de su subjetividad para ser una multiplicidad, son y existen en la medida en que lo hacen unos con otros: las ratas se vuelven infinitas porque ellos son una multiplicidad junto con ellas. De la misma manera, las personas que viven las guerras se transforman, devienen, hasta que forman una multiplicidad que lucha y que repele la peste. Sin embargo, esta repelencia no exime a los hombres "sanos", aquellos que no han sido tocados por la peste, de contagiarse también. Esto es porque el hombre que ha devenido-rata se ha transformado molecularmente, y así, cualquiera está en la condición de transformarse, de devenir-animal. Estos hombres han devenido-animales, se contagian porque forman múltiples devenires.

Al fin comprendí, por lo menos, que había sido yo también un apestado durante todos esos años en que con toda mi vida había creído luchar contra la peste. Comprendí que había contribuido a la muerte de miles de hombres, que

\footnotetext{
${ }^{2}$ El humanismo, o más claro, el existencialismo humanista que Camus desarrolló como filosofía relaciona lo absurdo de la existencia con una posibilidad de que este absurdismo o falta de sentido se canalice o neutralice a través de una humanización que lleve al ser humano a hacer el bien.

${ }^{3}$ Jean-Pierre Vernant, en Problèmes de la guerre en Grèce ancienne, Mouton, p. 15-16.
} 
incluso la había provocado, aceptando como buenos los principios y los actos que fatalmente la originaban. (La peste, p. 281)

En este diálogo de Tarrou, uno de los personajes que luchan contra la peste en la novela, ya no se habla de la peste, de la enfermedad; de repente se han diluido los límites de la ficción y la realidad ha aparecido bruscamente: así como sucede con la peste, la guerra también supone una enfermedad global; no existe la subjetividad, sino la multiplicidad, y en esta condición múltiple y heterogénea, todos pueden ser unos apestados. Hay un contagio. Los hombres que devienen-ratas no son únicamente aquellos que toman un arma en el campo de guerra, sino todos aquellos a los que la peste toca con su aire aniquilador. Camus da una importante reflexión a través de Tarrou: en una guerra, se deviene-rata con la violencia, pero también con la pasividad y con la ignorancia. Aquel que acepta la peste deviene-rata tanto como aquel que la ha provocado.

El Universo no funciona por filiación. Así pues, nosotros sólo decimos que los animales son manadas, y que las manadas se forman, se desarrollan y se transforman por contagio. Esas multiplicidades de términos heterogéneos, y de cofuncionamiento por contagio, entran en ciertos agenciamientos, y ahí es donde el hombre realiza sus devenires-animales. (Mil mesetas, p. 248)

Hay entonces un devenir-animal múltiple. Cuando un hombre deviene-animal, no lo hace únicamente él, sino que todos a su alrededor van contagiándose paulatinamente hasta formar un devenir múltiple. Los hombres en la novela forman manadas y la enfermedad de la peste se apropia del cuerpo, y el cuerpo contagiado no es más el cuerpo, la enfermedad ya no es la peste: el cuerpo en sí es la enfermedad. Cuando el cuerpo se ha contagiado con la peste, hay posibilidad de contagio con otros cuerpos: el cuerpo que repele es también el cuerpo que contagia. En Camus, los cuerpos enfermos son aislados; sin embargo, la enfermedad se propaga inevitablemente porque una vez que ha habido un solo devenir-rata, todos empiezan a dejar de ser hombres y pasan al plano del devenir-rata, porque se trata de una multiplicidad. El deveniranimal, el devenir-rata, en la guerra, es antídoto al mismo tiempo que es enfermedad.

Los hombres de Orán vieron llegar a la peste, a la guerra, y se han contagiado con la enfermedad y han devenido-animales al punto de que han formado una manada que contagia y que ya no es humana -tampoco es animal, solamente es-En la peste, en las guerras, los cuerpos son la materia que potencia la enfermedad y el contagio. Así, el devenir-animal no se detiene, no puede eliminarse:

Oyendo los gritos de alegría que subían de la ciudad, Rieux tenía presente que esta alegría está siempre amenazada. Pues sabía que esta muchedumbre dichosa ignoraba lo que se puede leer en los libros, que el bacilo de la peste no muere ni desaparece jamás. (La peste, p. 328)

Hay un devenir-animal cada vez que una guerra estalla -y se dice "cada vez" porque una guerra es del orden de la repetición diferente: siempre vuelve, se repite infinitamente, pero distinta-. Así concluye Camus el libro (véase primer epígrafe del trabajo), recordando al lector que la peste siempre vuelve inevitablemente. Cuando un hombre toma un arma y se va a la guerra, empieza su proceso de devenir, y cuando lo culmina, ya no es más un hombre, es un hombre que ha devenido-rata, que se ha transformada al punto de que ya no lleva consigo la vida, sino la peste: la guerra conlleva una transformación. 
Un devenir no es una correspondencia de relaciones. Pero tampoco es una semejanza, una imitación y, en última instancia, una identificación. Devenir no es progresar ni regresar según una serie. Y, sobre todo, devenir no se produce en la imaginación, incluso cuando ésta alcanza el nivel cósmico o dinámico. Los devenires animales no son sueños ni fantasmas. Son perfectamente reales. Pero, ¿de qué realidad se trata? Pues si devenir animal no consiste en hacer el animal o en imitarlo, también es evidente que el hombre no deviene "realmentell" animal, como tampoco el animal deviene realmente otra cosa. El devenir no produce otra cosa que sí mismo. (Mil mesetas, p. 244)

Así como lo explican Deleuze y Guattari, el hombre de la guerra, el guerrero, no involuciona (en el sentido de "regresión") hasta convertirse en una rata -de la misma manera en la que la rata no podría evolucionar hasta ser un hombre-. No se trata de eso el devenir, sino de la forma en que se establece una relación tal que un hombre puede transformarse en una rata. No es que en La peste los combatientes de guerra imiten a las ratas, sino que dentro de ellos ha habido una transformación que ha posibilitado una comunicación con otro: la rata es este otro. Hay una simbiosis entre los cuerpos.

El devenir siempre es de otro orden que el de la filiación. El devenir es del orden de la alianza. Si la evolución implica verdaderos devenires es en el basto dominio de las simbiosis que pone en juego seres de escalas y reinos completamente diferentes, sin ninguna filiación posible. (Mil mesetas, p. 244 y 245)

Hay entre el hombre y la rata una relación de simbiosis en la que ambos seres comparten intensidades y las comunican de un cuerpo a otro. Crean y establecen una zona de vecindad en la que ambos conviven y se relacionan. En esta zona de vecindad, el hombre no deja de ser hombre y la rata no deja de ser rata; ambos son ellos y son el otro al mismo tiempo.

La guerra y la peste parecen funcionar de la misma forma. La miseria que las dos conllevan es palpable en los ciudadanos de Orán. La peste es una novela realista que trabaja de manera simbólica; la guerra es una peste que puede azotar a cualquier ciudad, incluso a aquellas que han sido olvidadas, como Orán. Sin embargo, este simbolismo impregnado en la novela no implica que la peste o las ratas se reduzcan a simples metáforas: los hombres de La peste no son hombres ya, tampoco son ratas; lo que llega a la ciudad no es guerra, tampoco es una peste. Ninguno es totalmente lo que era inicialmente. Camus no establece una metáfora, o quizás establezca una que puede pensarse también como un devenir.

¿Por qué todo acontecimiento es del tipo de la peste, la guerra, la herida, la muerte? (Lógica del sentido, p. 110)

En esta cita de Lógica del sentido, Deleuze propone a los acontecimientos dentro de la dinámica de la guerra, de la peste, quizás porque -y como se ha analizado hasta ahora- estos generan un contagio. La guerra de Camus, entonces, no es otra cosa que un gran acontecimiento en el que se produce un contagio global; los acontecimientos suponen la convergencia de distintos devenires, formando así una multiplicidad de devenires que coexisten entre sí. Y no solamente en La peste, sino que todas las guerras suponen un acontecimiento que funcionará como escenario de los devenires.

Por otro lado, el hombre al devenir-animal, al devenir-rata, no lo hace sino a consecuencia de una fascinación por el otro ser, fascinación que se da no por la falta, sino por la empatía con el otro. Es así que el hombre no puede despreciar a la rata, no la repele, sino que la acepta, lo 
seduce transformarse y tomar un lugar de semejanza con ella. Un devenir no es posible si no se desarrolla un vínculo entre ambos seres, y este vínculo no es en ningún momento el del rechazo, sino más bien el del deseo: el hombre que resta de sí su condición de hombre -el N-1-, lo hace porque desea devenir menor, devenir-animal. Los hombres-ratas que llevan la peste a Orán han devenido-ratas por una fascinación por el otro, por las ratas y su peste.

No obstante, esto ocurre únicamente en $L a$ peste, sino que ha ocurrido en distintos momentos de la literatura. Resulta entonces interesante pensar en Maus: relato de superviviente (1991), de Art Spiegelman, una novela gráfica donde las víctimas del holocausto nazi -aunque también los propios victimarios- son ratas. Y no porque realmente lo sean, sino porque han adquirido comportamientos y son víctimas de un trato que los ha hecho devenir-animales. Así explica Spiegelman en el epígrafe del libro, donde cita a Hitler y dice: «Los judíos son indudablemente una raza, pero no son humanos». Pareciera que Maus se desarrolla en un sentido contrario al de La peste -argumento que resulta válido en primeras instancias-, pues en este cómic son las víctimas las que se han transformado; sin embargo, si se habla de una enfermedad colectiva, una multiplicidad, es evidente que el devenir-rata se da posteriormente en ambos lados, tanto en los guerreros como en las víctimas. De esta manera, el devenir-animal es un resultado, un efecto, de la guerra: es la guerra la que propicia el escenario para que ocurra un devenir. La literatura parece ser así un espejo que muestra una realidad en la que los devenires-animales son posibles y son una consecuencia de un acontecimiento.

Siempre hay un hombre fascinado por dejar de ser él mismo, deseoso de la idea de ser otro para rearfirmarse a sí mismo y al resto. Esta fascinación se convierte en devenir: una desterritorialización del ser hasta que este deviene-rata. La peste supone así una lectura de la humanización, pero también de la deshumanización. En el caso de Orán, la guerra es el escenario del devenir-animal, del devenir-rata en los hombres; de la misma manera, esta guerra, que no es otra que la peste, existe permanentemente y puede llegar a cualquier ciudad: la peste existe porque existen hombres deseosos de devenir-animales, de devenir-ratas y de contagiar al otro con su devenir.

Esta dinámica de deshumanización que conecta a los hombres de Orán con un otro animal, un otro rata, nos hace pensar en nuestras propias guerras, es decir, en nuestras pestes y en nuestros devenires-ratas: La peste es un relato que nos ubica frente al espejo de nuestro propio contagio colectivo.

\section{Referencias}

CAMUS, Albert. La peste. Quito: Libresa, 2013.

DELEUZE, Gilles; GUATTARI, Félix. Mil mesetas: Capitalismo y Esquizofrenia. Valencia: Pre-Textos, 2004.

SPIEGELMAN, Art. Maus: relato de un superviviente. Barcelona: Penguin Random House, 2018. 